\title{
Data-driven Public Health and Social Intervention Modeling to Promote Effective Policy Responses to Curtailing the COVID- 19 Pandemic
}

This paper was downloaded from TechRxiv (https://www.techrxiv.org).

\section{LICENSE}

CC BY 4.0

SUBMISSION DATE / POSTED DATE

06-07-2020 / 08-07-2020

\section{CITATION}

Qiu, Robin (2020): Data-driven Public Health and Social Intervention Modeling to Promote Effective Policy Responses to Curtailing the COVID-19 Pandemic. TechRxiv. Preprint.

https://doi.org/10.36227/techrxiv.12616142.v1

\section{$\mathrm{DOI}$}




\title{
Data-driven Public Health and Social Intervention Modeling to Promote Effective Policy Responses to Curtailing the COVID-19 Pandemic
}

\author{
Robin G. Qiu \\ Penn State \\ robinqiu@psu.edu
}

\begin{abstract}
We might have to live with COVID-19 until 2025 according to a recent report published in Science! But if we act smartly, the adverse consequence of living with the virus could be minimized. Currently, many states in the United States are seeing spiking cases on a daily basis due to their inadequate policy responses to COVID-19. This paper promotes more research on improving SEIR modeling as it will play a critical role in facilitating the decisionmaking on promoting and implementing appropriate public health and social interventions. Hopefully, policymakers will listen to science and enact and implement adequate policy responses in combating the COVID-19 pandemic in each of the states across the United States so that we can win this "war" and be well prepared for the promising future.
\end{abstract}

Keywords: COVID-19, Public Health and Social Intervention, Mitigation Measure, SEIR Modeling, Policy Response, Machine Learning

\section{Introduction}

The novel coronavirus SARS-CoV-2 or COVID-19 pandemic has caused painful social and economic disruption around the world, including the largest global recession since the Great Depression. Fighting to contain the COVID-19 pandemic has become the most important and challenging task worldwide. Before a COVID-19 vaccine or effective treatment becomes widely available, experts in epidemiology, medicine, and public health have warned and suggests that public health and social interventions, like social distancing, travel restriction, gathering ban, face covering mandate, and stay-at-home or shelter-in-place lockdown, as non-pharmaceutical mitigation measures would be the choices of "weapons" to fight COVID-19.

Promisingly, Dehning et al. [1] reveal that prompt and appropriate public health interventions are effective in containing the COVID-19 pandemic recently in Germany. In the US, policy response to taming the outbreak in the US has an exemplary evidence too. The northeast corridor centered at New York city quickly became the first epidemic center in the US due to the lack or failure of public health preparedness at the federal level when the COVID-19 started spreading in early March of 2020. It is noteworthy that this first epidemic in the US has got well curtailed over last 3 months or so due to the enacted public health intervention polices and placed corresponding mitigation measures at the state level across the region.

As of today, the $4^{\text {th }}$ of July 2020, however, the outcomes of fighting COVID-19 across the US are substantially different from state to state. Many states in the US have been struggling in containing the spread of COVID-19. Sunbelt's states have been particularly hit hard as new cases are spiking and keeping resetting their daily new records for weeks. For example, Florida shattered a single-day record, topping 11,000 new cases on Independence Day. The latest data clearly shows that COVID-19 hospitalizations have begun to swell in states like Texas, Arizona, Florida, and California. 
The rising cases across the US are intuitively attributed to "knucklehead" behavior by Americans who refuse wearing masks or obeying other mitigation rules. The failure of promptly enacting and strictly implementing public health intervention policies at both the federal and the state levels can be the real contributor to this unfortunate consequence. It is thus extremely urgent for us to investigate a data-driven approach that can real-time and dynamically assess and model the evolution of the pandemic at different levels, aimed at providing policymakers with quantified consequences of different mitigation policies in scientific rigor [2].

In this paper, we present a potential data-driven framework for facilitating policymaking on the timing and restrictive levels of public health and social interventions at the state level as time goes. The remainder of this paper is organized as follows. Section 2 shows the need for the stated framework using a lesson study learned from mistakes. Section 3 then presents a possible datadriven model, which can be utilized to develop appropriate pandemic response policies at the state level. At last, final remarks of this paper will be provided.

\section{The Need for Data-driven Health Intervention Policy Modeling}

The compartmental SEIR (Susceptible, Exposed, Infectious, and Recovered) model or the like has been well utilized to model the spread of an infectious disease for decades (Fig. 1). SEIR proves effective in facilitating decision-making in developing and monitoring health intervention policies to fight an endemic, epidemic, or pandemic. However, the notorious reproduction number $\left(\mathrm{R}_{0}\right)$, an epidemiologic metric used to describe the contagiousness or transmissibility of infectious agents, in an SEIR model has never been easy to get accurately estimated due to the complexity of measuring and estimating $\mathrm{R}_{0}[1]$. $\mathrm{R}_{0}$ is contextual, a function of $\lambda, \delta$, and $\mu$.

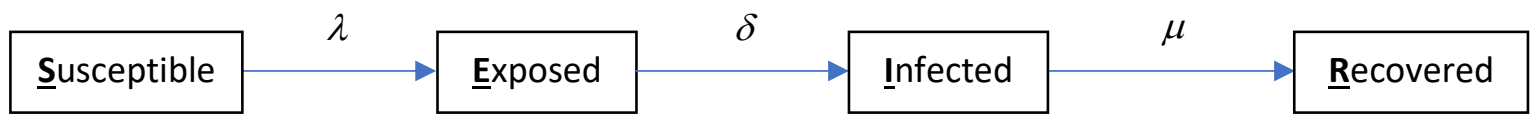

Fig. 1. An SEIR model - $\lambda$ : expected number of persons an infected person infects per day, $\delta: 1 /$ (days to get infected, also called incubation time), $\mu: 1 /($ days to recover).
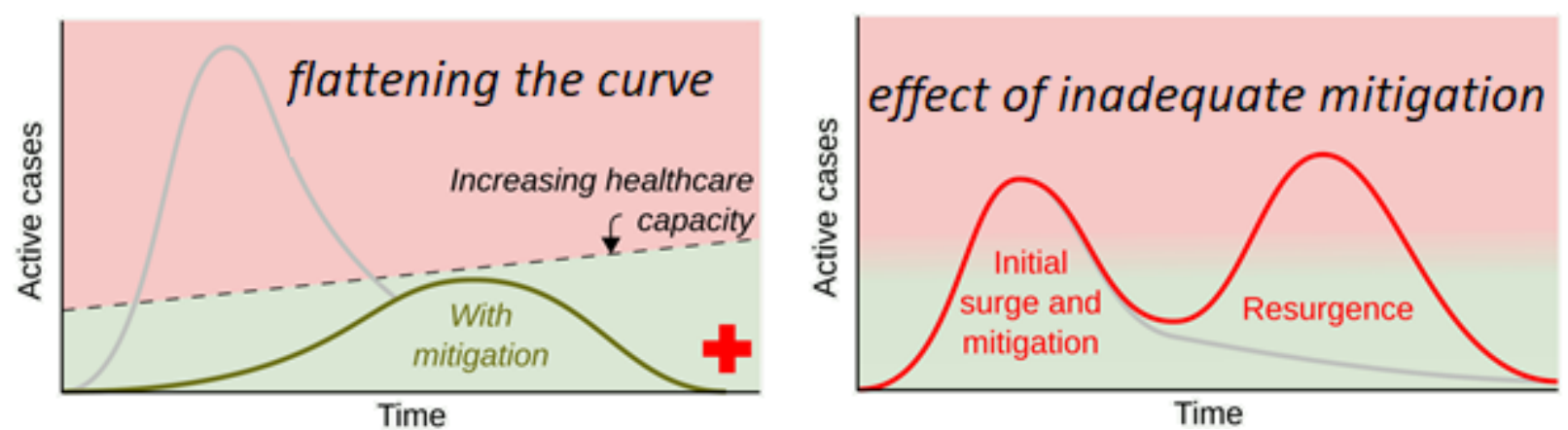

Fig. 2. Theoretical active cases of a pandemic over time 
In the US, flattening the case curve with appropriate mitigation while increasing healthcare capacity over time has become the essential strategy of every state across the country (shown in the left side of Fig 2.). We know that inadequate mitigation could result in a resurgence regionally and nationally (shown in the right side of Fig. 2). $\mathrm{R}_{0}$ dictates the curve shape in Fig. 2.

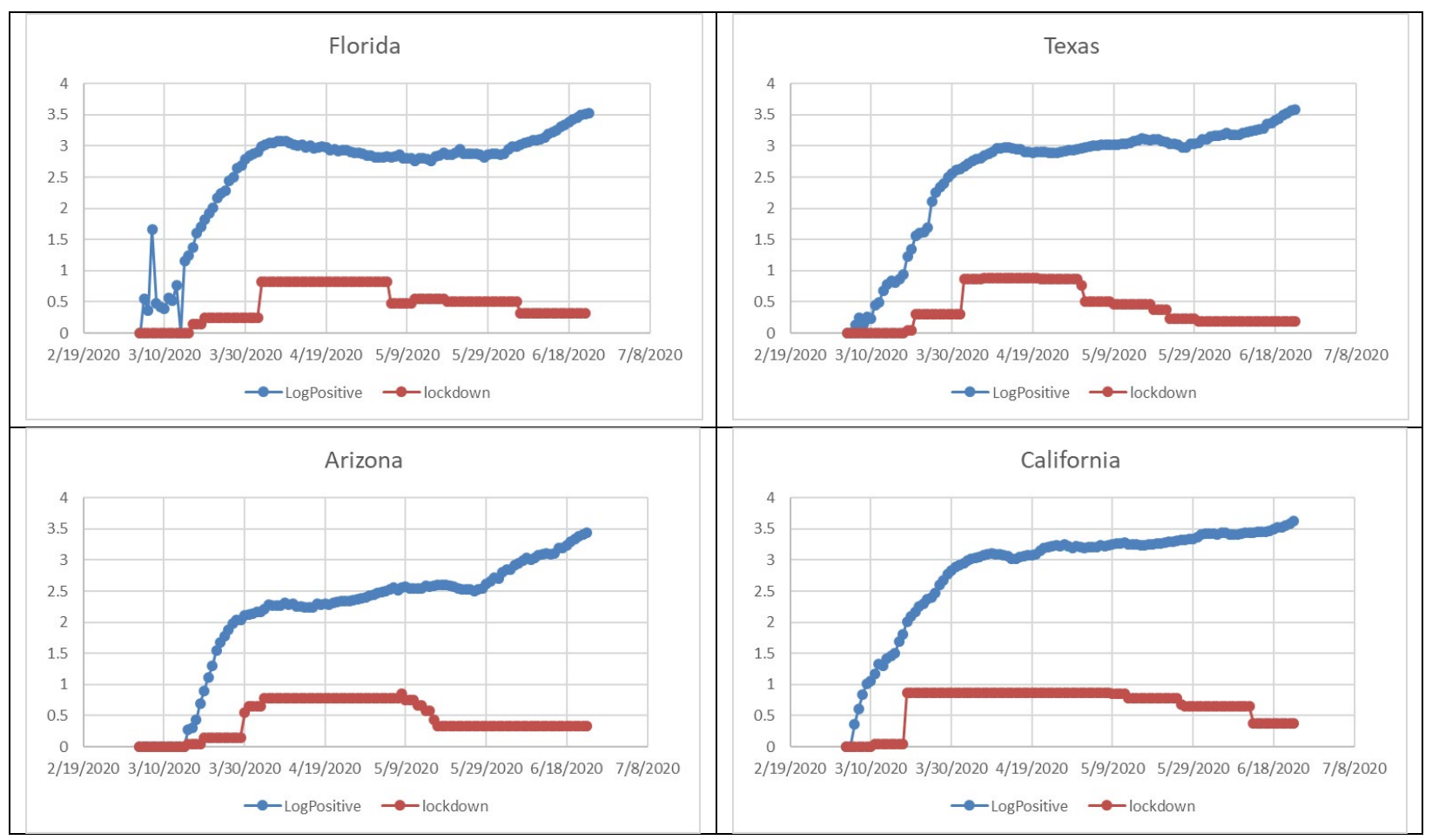

Fig. 3. Daily cases vs. mitigation measures in 4 sunbelt states from March 1 to June 23, 2020

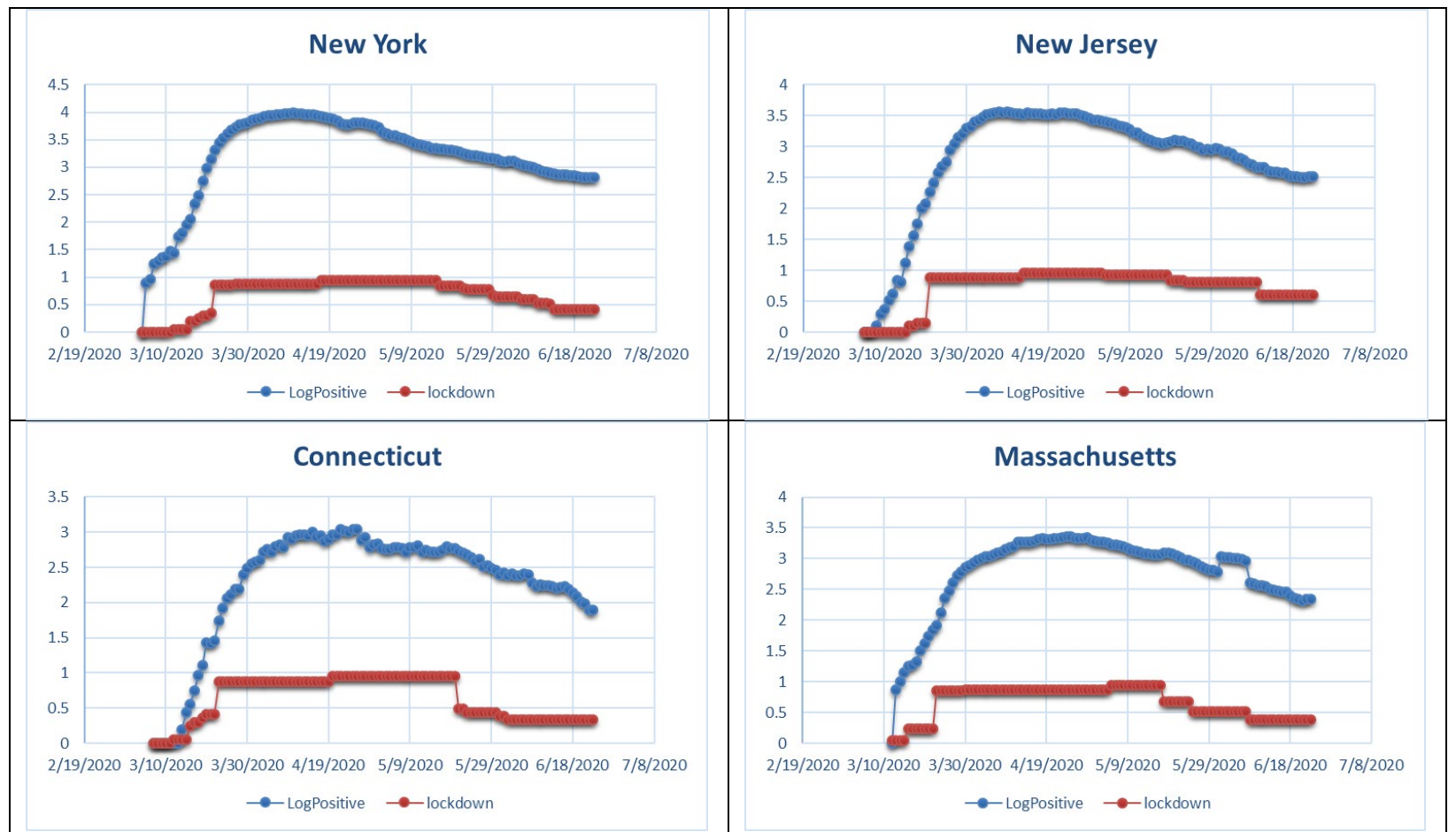

Fig. 4. Daily cases vs. mitigation measures in 4 Northeast states from March 1 to June 23, 2020 
As discussed earlier, inadequate mitigation could result in a resurgence regionally and nationally as shown in the right side of Fig. 2. This is exactly what is happening in the US where public health preparedness and policy enactment and enforcement in fighting the COVID-19 pandemic failed big time at both the federal and some state levels. As of June 23, 2020, the outcomes of fighting COVID-19 across the US are substantially different from state to state as witnessed since the beginning of March 2020 when the outbreak started in the US (Fig. 3 and Fig. 4).

Data never lies. As example, Fig. 3 and Fig. 4 show daily cases in all 8 states dropped considerably after adequate public health and social intervention policies were enacted and enforced at the very beginning [6]. However, some governors reopened their states too soon without following the reopening guidance provided by CDC. As expected, the resurgence becomes inevitable, which is evidenced by the fact as shown in Fig. 3.

From Fig. 3 and Fig. 4, we can clearly uncover that all 4 sunbelt states never implemented the most stringent mitigation measures [6] compared to these 4 northeast states. In fact, the restriction level of public health and social intervention was increased in these 4 northeast states when the necessity was identified and justified. The similar pattern can be identified from Google community mobility data as shown in Fig. 5 and Fig. 6 [7]. In every state across the US, community moving around and corresponding mobility changing pattern at the state level are highly correlated to the mitigation measures adopted and enforced in the state respectively [6]. Due to societal and economic considerations and sometimes individual beliefs or political stance, all 4 sunbelt's states lessened the COVID-19 restrictions too soon and too fast. The adverse consequence surfaced.

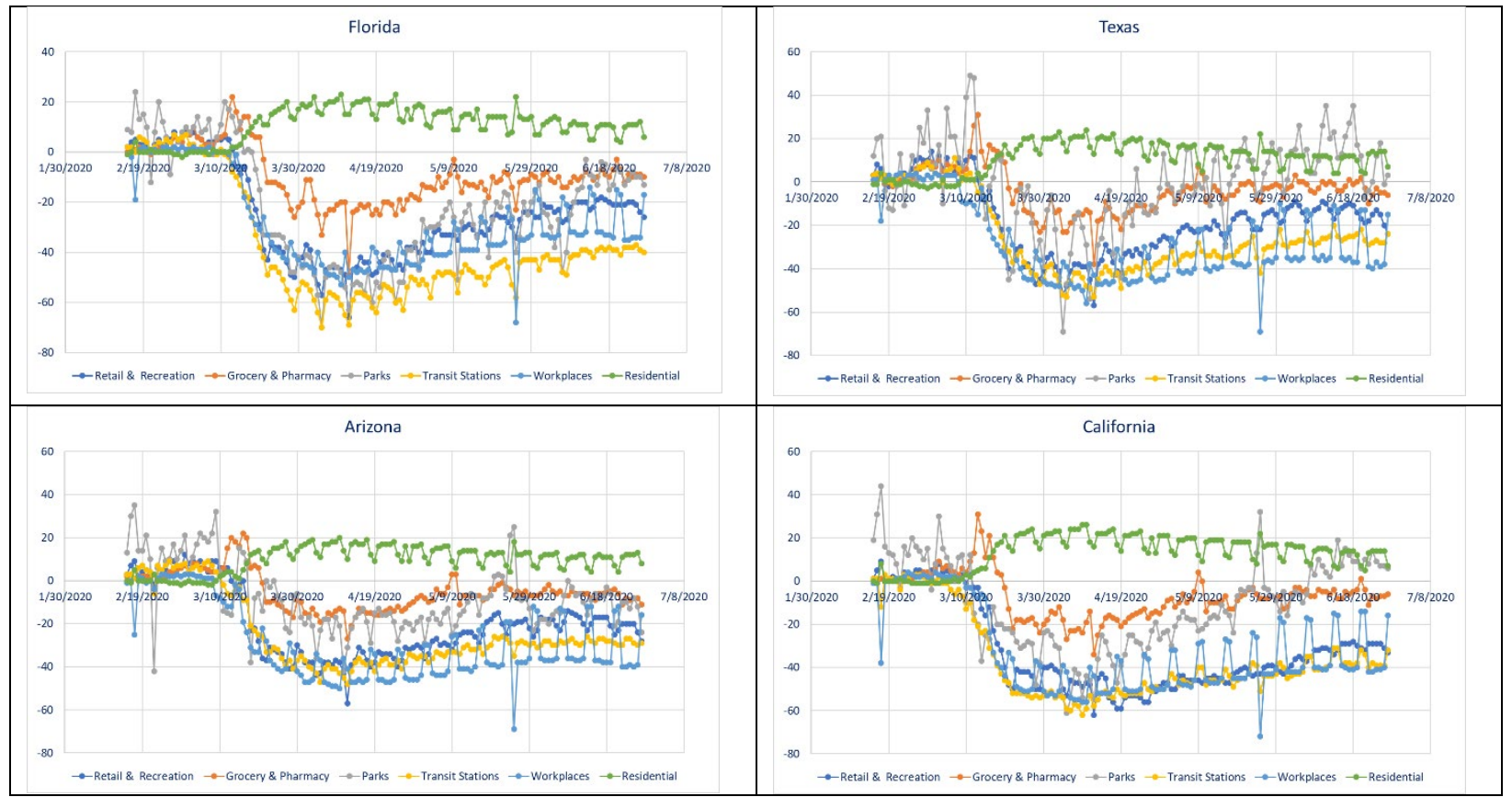

Fig. 5.4 sunbelt states' daily mobility change from Feb 151 to June 27, 2020

Currently, daily cases in the sunbelt states keep spiking, which has raised fears that many other states could follow sunbelt states' footsteps. Because of the lack of adequate and systemic 
federal-level policies and strict enforcement across the US, epidemiologists are afraid that people from the South and West could spread the virus to other regions where daily cases are dropping. Unless all the states across the US act together effectively and holistically, the US will be at risk of confronting repeated waves of the COVID-19 pandemic for many years to come. Therefore, there is an imminent need for a real-time data-driven framework that can facilitate policymaking on the timing and mitigation at the state level across the country.

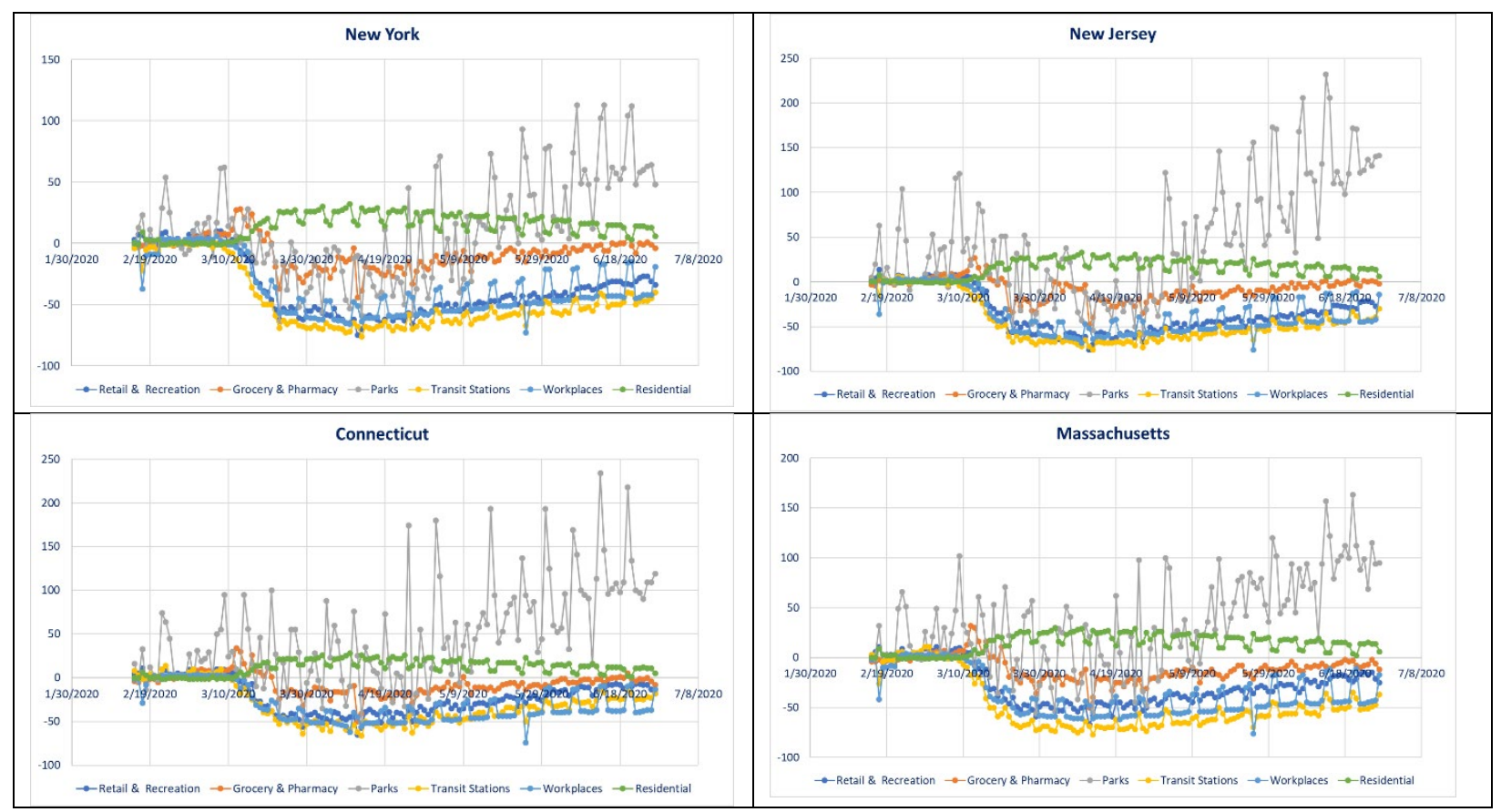

Fig. 6.4 northeast states' daily mobility change from Feb 151 to June 27, 2020

\section{Promoting and Developing Real-Time Data-driven Policy Response Model}

Developing SEIR or the like models that can help develop adequate intervention and mitigation measures in fighting a pandemic is never easy but challenging. This is particularly true for COVID-19 as it is evolving. Although there is still too much unknown, a lot of lessons have been learned since its outbreaks in China, South Korea, Japan, and Europe [7-8]. Given the results from Dehning et al. [1] and Lika et al. [3], we believe that more research on improving SEIR modeling will play a critical role in facilitating the decision-making on public health and social interventions, which can surely help state policymakers enact and implement state policy responses with scientific rigor in combating the COVID-19 pandemic in each of the states across the United States.

Public health and social interventions mainly include not limited to school closure, social distancing, travel restriction, gathering ban, face covering mandate, and stay-at-home or shelterin-place lockdown. Enacting and enforcing state policy responses to COVID-19 can be contextual, sophisticated, and time-dependent. As a result, policymaking is particularly challenging when COVID-19 was at its early stage of outbreaks due to its novelty with too much unknown and lacking in-person combating experience. As evidenced, New York city became the 
epidemic enter in the US was largely due to the early mistake of delaying school closures and city shutdown in the middle March 2020. Fortunately, New Your state learned the lesson and enacted and implemented stringent mitigation measures to contain the spread of COVID-19 in late March 2020. As witnessed, the adopted policy response proves very effective.

In fact, many lessons could be learned from China, South Korea, Japan, and Europe [7-8]. However, easier said than done. The situations in some states become even worse when their policymakers respond to COVID-19 by taking a partisan stance rather than listening to science. For example, as more and more studies [8-11] reveal COVID-19 can be transmitted before symptom onset, community transmission can be reduced if everyone wears face coverings. This is exceptionally critical and necessary for people who have been infected but are asymptomatic and contagious [12-13]. Although experts and political leaders throughout the United States have been urging Americans to wear face coverings in public, some states still refused enacting and implementing adequate public health and social interventions by including this essential mitigation measure, resulting in a surge in coronavirus cases across much of the country.

We think that policymakers who tend to be influenced by their partisan stance rather than science and the reality is mainly due to the lack of the needed real-time, data-driven, and interactive policymaking reference tools [2]. As discussed earlier, we believe that improved SEIR modeling will play a critical role in facilitating the decision-making on public health and social interventions at the state level (Fig. 7). Studies confirm that mitigation measures have been the greatest "weapons" to fight COVID-19 effectively $[1,3,14]$. More importantly, they provide the differences, time, and rates of dropping the reproduction number $\mathrm{R}_{0}$ that had been made due to different levels of mitigation measures that have been enforced over their study periods.

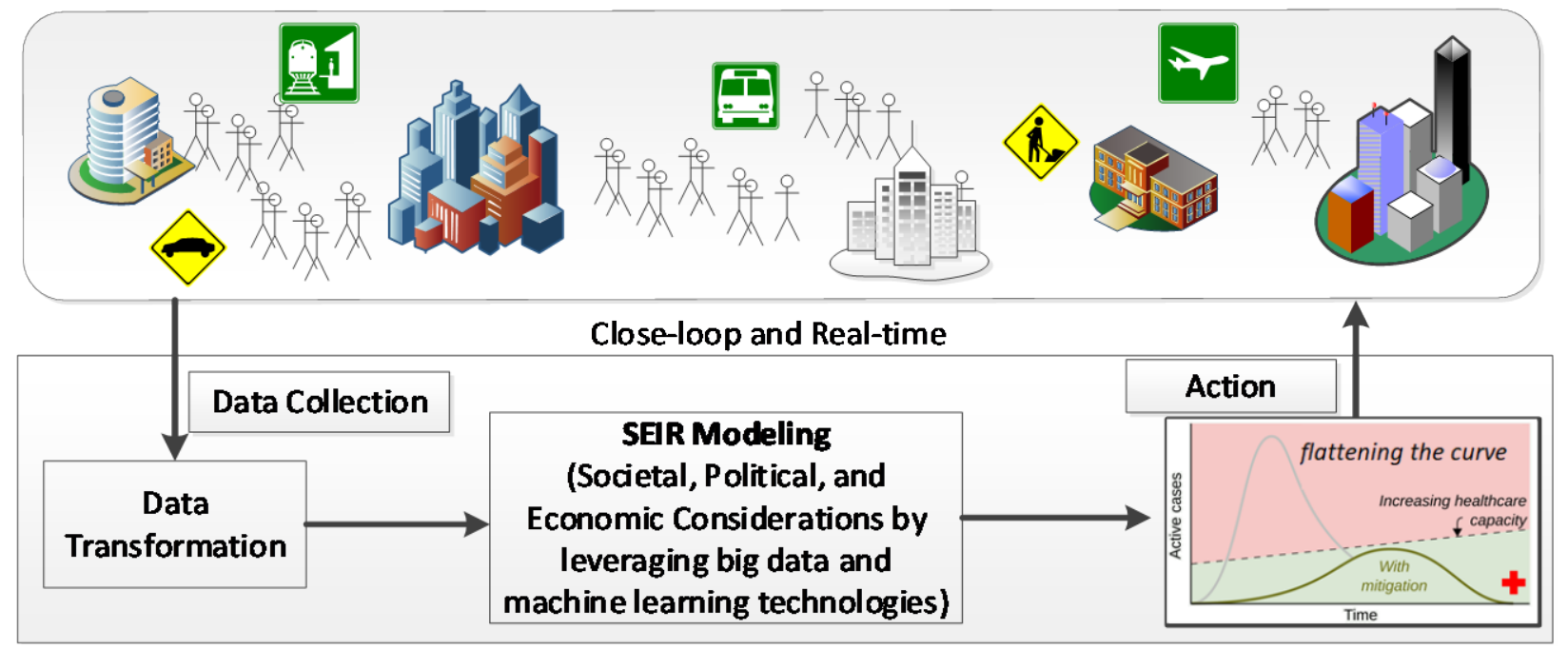

Fig. 7. Real-time data-driven interactive tool to facilitate policymaking in combating COVID-19

As known, $\mathrm{R}_{0}$ dictates the curve shape of a pandemic, while public health interventions determine the change of $\mathrm{R}_{0}$ over time, i.e., when and how much $\mathrm{R}_{0}$ rises or drops. It is thus extremely urgent for us to investigate a data-driven approach that can real-time and dynamically estimate time-dependent $\mathrm{R}_{0}$ and other parameters in facilitating the construction of an SEIR 
model for each state in the US. As a result, effective and proactive rather than reactive policy responses can be enacted and enforced promptly $[15,16]$. Undoubtedly, an interactive tool can help policymakers well understand the quantified rather than qualified consequences of different state-level mitigation policies in a scientific and real-time manner $[2,17]$. To make such policy response tool more realistic and applicable, more importantly acceptable, the capacity of healthcare systems and asymptomatic transmission must be included in the SEIR model (Fig. 8).

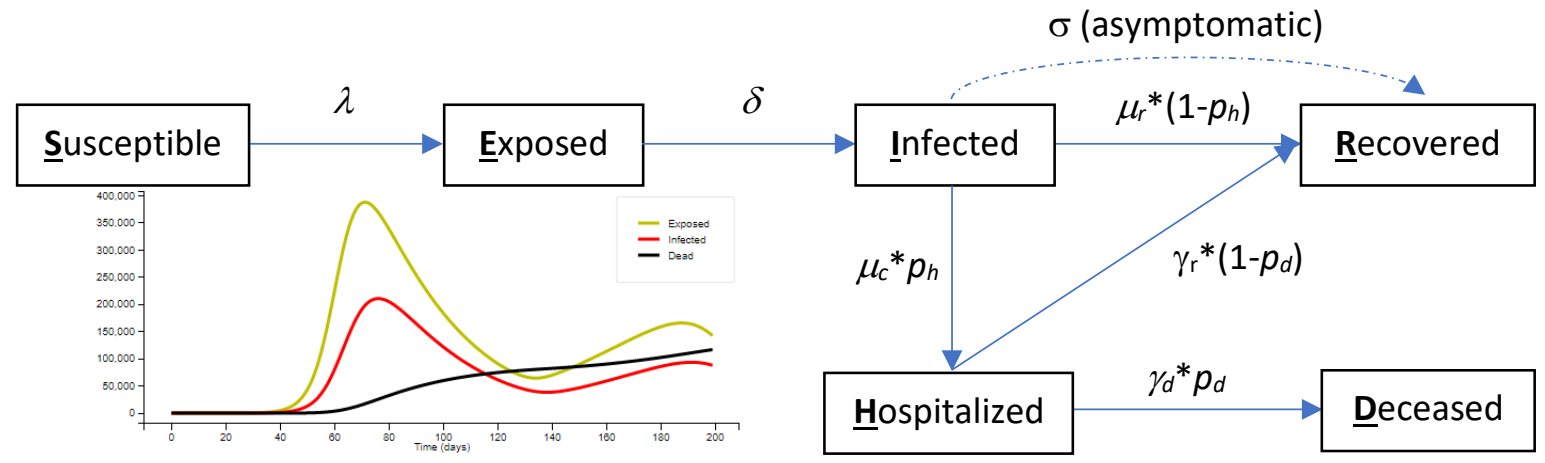

Fig. 8. An Extended SEIR model ( $\lambda$ : expected number of persons an infected person infects per day, $\delta: 1 /$ days to get infected, also called incubation time, $\sigma: 1 /$ days to recover without testing and treatment, $\mu_{r}: 1 /$ days to recover without hospitalization, $\mu_{c}: 1 /$ days to be hospitalized from infection, $p_{h}$ : percentage of Infected persons to be hospitalized, $\gamma_{r}: 1 /$ days to recover from hospitalization, $p_{d}$ : percentage of Infected persons to die, $\gamma_{d}$ : 1 /days of hospitalized patients to die. $\mathrm{R}_{0}$ is contextual, a function of time and these above-mentioned parameters.)

As the COVID-19 pandemic data is daily updated in the United States, we can leverage the currently available rich COVID-19 pandemic data and machine learning algorithms [18] to extend and enhance SEIR modeling with parameter auto-adjustment capability in real time (Fig. 7). The key features of this interactive tool should include at least the following:

- The effects of public health interventions can be viewed at the state level. In the long run, it should be drilled down to a community level.

- For a proposed mitigation measure, quantified estimates of related parameters in SEIR modeling can be calculated with scientific rigor. For example, the contact rate at which an infectious individual comes into contact and infects others, is not constant, but modulated by social behavior and policy responses. Political mitigation strategies to reduce the effective reproduction number has a time delay (e.g., two weeks). As an SEIR model is contextual and time-dependent, parameters must be able to be learned and automatically adjusted as time goes.

\section{Final Remarks}

In summary, different from the early stage of SEIR modeling in which predictions were used as a general reference for decision-making at either the state or the national levels, the proposed 
SEIR model promotes its practical capabilities of helping fight the COVID-19 pandemic by focusing on assessing and monitoring policy responses. As discussed earlier, by leveraging the available rich pandemic data and machine learning algorithms, the developed model can capture the reality and predict the trend in real-time and with a significantly improved accuracy, facilitating decision-making in enacting and enforcing adequate policy responses to combating the COVID-19 pandemic.

Note that the proposed model can be promisingly deployed as an interactive tool for serevral states to coordinate their efforts of understanding and controlling the trend of COVID-19 regionally. If data at local community levels becomes available, the proposed approach can be easily customized at a more granular level. Moreover, the developed approach can be easily customized and adopted, locally, regionally, nationally, and internationally.

We might have to live with COVID-19 for years to come [19]! But if we act smartly, the adverse consequence of living with the virus could be minimized.

\section{References}

1. J. Dehning, J. Zierenberg, F.P. Spitzner, M. Wibral, J.P. Neto, M. Wilczek, and V. Priesemann. Inferring change points in the spread of COVID-19 reveals the effectiveness of interventions. Science https://doi.org/10.1126/science.abb9789 (2020).

2. R.G. Qiu. Leveraging Rich Data and Machine Learning to Facilitate Policy Making on Public Health Interventions in Fighting to Contain the COVID-19 Pandemic. TechRxiv. Preprint. https://doi.org/10.36227/techrxiv.12573755.v1 (2020).

3. K. Linka, M. Peirlinck, E. Kuhl. The reproduction number of COVID-19 and its correlation with public health interventions. medRxiv (2020). May 10, 2020: https://doi.org/10.1101/2020.05.01.20088047.

4. R.M. Anderson, H. Heesterbeek, D. Klinkenberg, and T.D. Hollingsworth. How will country-based mitigation measures influence the course of the COVID-19 epidemic?. The Lancet, 395(10228), pp.931-934 (2020).

5. M. Enserink, K. Kupferschmidt. With COVID-19, modeling takes on life and death importance. Science, 367, pp.1414-1415 (2020).

6. R.G. Qiu, E. Wang, and J. Qiu. Development of Data-driven Models to Facilitate Policymaking in Response to Containing the COVID-19 Pandemic. Submitted to 2020 INFORMS Service Science, Dec. 19-21, 2020.

7. Google COVID-19 Mobility Reports, https://www.google.com/covid19/mobility/.

8. S.E. Eikenberry, M. Mancuso, E. Iboi, T. Phan, K. Eikenberry, Y. Kuang, E. Kostelich, and A.B. Gumel. To mask or not to mask: Modeling the potential for face mask use by the general public to curtail the COVID-19 pandemic. Infectious Disease Modelling. (2020)

9. S. Feng, C. Shen, N. Xia, W. Song, M. Fan, and B. J. Cowling. Rational use of face masks in the COVID-19 pandemic. The Lancet Respiratory Medicine, 8(5), pp. 434-436 (2020).

10. T. Greenhalgh, M.B. Schmid, T. Czypionka, D. Bassler, and L. Gruer. Face masks for the public during the covid-19 crisis. BMJ, 369 (2020). 
11. V.C. Cheng, B. C. Wong, V. W. Chuang, S. Y., So, J. H. Chen, S. Sridhar, ... \& K. Y. Yuen. The role of community-wide wearing of face mask for control of coronavirus disease 2019 (COVID-19) epidemic due to SARS-CoV-2. Journal of Infection, 81, pp. 107-114 (2020)

12. Y. Bai, L. Yao, T. Wei, F. Tian, D. Y. Jin, L. Chen, and M. Wang. Presumed asymptomatic carrier transmission of COVID-19. Jama, 323(14), pp. 1406-1407 (2020).

13. M. Day. Covid-19: identifying and isolating asymptomatic people helped eliminate virus in Italian village. BMJ: British Medical Journal (Online), 368 (2020).

14. H. Sjödin, A. Wilder-Smith, S. Osman, Z. Farooq, and J. Rocklöv. Only strict quarantine measures can curb the coronavirus disease (COVID-19) outbreak in Italy, 2020. Eurosurveillance, 25(13), 2000280, (2020)

15. B. J. Cowling, and A. E. Aiello. Public health measures to slow community spread of coronavirus disease 2019. The Journal of infectious diseases, 221(11), pp. 1749-1751 (2020).

16. A. Goodman-Bacon, and J. Marcus. Using difference-in-differences to identify causal effects of covid-19 policies. Discussion Papers of DIW Berlin (2020)

17. R. G. Qiu, Service science: The foundations of service engineering and management. John Wiley \& Sons (2014).

18. S. Latif, M. Usman, S. Manzoor, W. Iqbal, J. Qadir, G. Tyson, ... and J. Crowcroft. Leveraging Data Science To Combat COVID-19: A Comprehensive Review. TechRxiv. Preprint. https://doi.org/10.36227/techrxiv.12212516.v1(2020).

19. S. M. Kissler, C. Tedijanto, E. Goldstein, Y. H. Grad, and M. Lipsitch. Projecting the transmission dynamics of SARS-CoV-2 through the postpandemic period. Science, 368(6493), pp. 860-868 (2020). 